\title{
DualityTheorem Over the Cone of Monotone Functions and Sequences in Higher Dimensions
}

\author{
SORINA BARZA ${ }^{a}$, HANS P. HEINIG $^{b, *}$ \\ and LARS-ERIK PERSSON ${ }^{a, \dagger}$
}

aDepartment of Mathematics, Luleå University, S-97187 Luleå, Sweden; ${ }^{\mathrm{b}}$ Department of Mathematics and Statistics, McMaster University, Hamilton, Canada, L8S 4K1

(Received 25 April 2000; In final form 10 August 2000)

Let $f$ be a non-negative function defined on $\mathbb{R}_{+}^{n}$ which is monotone in each variable separately. If $1<p<\infty, g \geq 0$ and $v$ a product weight function, then equivalent expressions for

$$
\sup \frac{\int_{\mathbb{R}_{+}^{n}} f g}{\left(\mathbb{R}_{+}^{n} f^{p} v\right)^{1 / p}}
$$

are given, where the supremum is taken over all such functions $f$.

Variants of such duality results involving sequences are also given. Applications involving weight characterizations for which operators defined on such functions (sequences) are bounded in weighted Lebesgue (sequence) spaces are also pointed out.

Keywords and Phrases: Duality theorems; Monotone functions; Multidimensional functions; Weighted inequalities

1991 Mathematics Subject Classifications: Primary: 26D15, 47B38; Secondary: 42B20, 46E30

* Research supported by NSERC grant A-4837.

${ }^{\dagger}$ Corresponding author.

ISSN: 1025-5834. Online ISSN: 1029-242X. (C) 2002 Taylor \& Francis Ltd DOI: $10.1080 / 10755830290013416$ 


\section{INTRODUCTION}

If $0 \leq f$ is a decreasing ( $\equiv$ non-increasing) function of a single variable, then Sawyer [8] established an explicit form of an $L^{p}$-duality theorem for such functions, and applied it to prove weight characterizations for which the identity operator, the Hardy integral operator and its conjugate defined on monotone functions is bounded on weighted Lebesgue spaces. As a consequence weight characterizations were obtained for which a number of classical operators, such as the HardyLittlewood maximal function and the Hilbert transform, are bounded on weighted Lorentz spaces.

It is natural to seek extensions of such duality theorems to functions of several variables monotone in each of its variables separately. Such generalizations were recently obtained by Barza, Persson and Stepanov [3]. To explain some of their generalizations we require some notation: We write $\mathbb{R}_{+}^{n}=\left\{\left(x_{1}, \ldots, x_{n}\right): x_{i} \geq 0, i=1,2, \ldots, n\right\}$ and $\mathbb{R}_{+}^{1}=\mathbb{R}_{+}$. If $f: \mathbb{R}_{+}^{n} \rightarrow \mathbb{R}_{+}$is decreasing (increasing) separately in each variable we write, $0 \leq f \Downarrow(0 \leq f \Uparrow)$. A set $D \subset \mathbb{R}_{+}^{n}$ is said to be decreasing if its characteristic function $\chi_{D} \Downarrow$, and clearly if $0 \leq h \Downarrow$ and $t>0$, then the set $D_{h, t}=\left\{x \in \mathbb{R}_{+}^{n}: h(x)>t\right\}$ is decreasing.

If $0<q<p<\infty,(1 / r)=(1 / q)-(1 / p)$, then it was shown in [3] that

$$
\begin{aligned}
\sup _{0 \leq f \Downarrow} \frac{\left(\int_{\mathbb{R}_{+}^{n}} f^{q} u\right)^{1 / q}}{\left(\int_{\mathbb{R}_{+}^{n}} f^{p} v\right)^{1 / p}} \approx & \frac{\left(\int_{\mathbb{R}_{+}^{n}} u\right)^{1 / q}}{\left(\int_{\mathbb{R}_{+}^{n}} v\right)^{1 / p}} \\
& +\sup _{0 \leq h \Downarrow}\left\{\int_{0}^{\infty}\left(\int_{D_{h, t}} u\right)^{r / q} d\left(\int_{D_{h, t}} v\right)^{-r / p}\right\}^{1 / p},
\end{aligned}
$$

where $u$ and $v$ are non-negative (weight) functions. For the case $0<p \leq q<\infty c f$. [2].

If $q=1<p<\infty$ and $u=g \geq 0$ then (1.1) with $n=1$ is a variant of the duality theorem given in [8]. Now, if $n>1$ and $0 \leq h \Downarrow$ is arbitrary, then a quantitative form of the sets $D_{h, t}$ may be difficult to give and this limits the applicability of the duality formula (1.1).

The purpose of this paper is to prove a duality formula of the form (1.1) with $q=1, u=g$ and $v$ a product weight, that is, a weight function of the form $v(x)=v\left(x_{1}, \ldots, x_{n}\right)=v_{1}\left(x_{1}\right) \cdots v_{n}\left(x_{n}\right), v_{i} \geq 0, i=1, \ldots, n$. This permits explicit applications to mapping properties of operators 
defined on the cone of such monotone functions between weighted Lebesgue spaces. We illustrate this for the identity operator on $\mathbb{R}_{+}^{n}$, (a result also proved in [2]) and in case $n=2$ by the Hardy integral operator defined on function decreasing in both variables separately. Also, a corresponding discrete version of the duality principle is given where the functions are replaced by sequences $\left\{a_{i j}\right\}_{i, j=1}^{\infty}$, which are decreasing in both indices. Again an application is given for the Césaro operator defined on such monotone sequences. The case of sequences monotone in a single index was proved in [7] under slight additional conditions on the weight sequence. In the proof given here (which also carries over to the one dimensional case) this additional assumption is not required.

The manuscript is structured as follows: The next section contains a number of known or easily derived results required. These include the weighted Hardy inequality and its discrete analogue in higher dimensions. In Section 3 the duality theorems for functions of several variables are given, while Section 4 contains the discrete analogue in two dimensions. The final section contains applications, that is, embedding theorems for the identity and Hardy operator defined on decreasing functions and a corresponding result for sequences of monotone type.

Throughout, functions are assumed to be measurable, constants, denoted by $A, B, C$, sometimes with subscripts, are always positive and may be different at different places. If $(X, \nu)$ is a measure space, then $L_{\nu}^{p}=L_{\nu}^{p}(X)=\left\{f\right.$ on $\left.X:\left(\int_{X}|f|^{p} d \nu\right)^{1 / p}<\infty\right\}, 1 \leq p<\infty$, and if $d \nu=$ $v(x) d x$ we write $L_{v}^{p}$. If $d \nu$ is the counting measure we obtain the sequence spaces $\ell_{\nu}^{p} . X$ is usually $\mathbb{R}_{+}^{n}$ or $\mathbb{N} \times \mathbb{N}$. The conjugate index of $p$ is $p^{\prime}=p /(p-1), p \neq 1$. Inequalities (such as (2.1)) are interpreted to mean that if the right hand side is finite, so is the left hand side and the inequality holds. The symbol $\approx(c f .(1.1))$ means that the quotient of the right and left hand side is bounded above and below by positive constants, while expressions of the form $0 \cdot \infty=\infty \cdot 0$ are taken as zero. Finally, if $x \in \mathbb{R}_{+}^{n}$ then $f(1 / x)=f\left(1 / x_{1}, \ldots, 1 / x_{n}\right), x^{\alpha}=x_{1}^{\alpha} \cdots x_{n}^{\alpha}$, $\alpha \in \mathbb{R}$ and

$$
\int_{x}^{\infty}=\int_{x_{1}}^{\infty} \cdots \int_{x_{n}}^{\infty} ; \int_{0}^{x}=\int_{0}^{x_{1}} \cdots \int_{0}^{x_{n}} .
$$

Other notations will be introduced when required. 


\section{PRELIMINARY RESULTS}

Our first result is the following higher dimensional Hardy inequality for product weights.

THEOREM 2.1 If $u$ and $v$ are product weights on $\mathbb{R}_{+}^{n}$ whose components are $u_{i}, v_{i}, i=1, \ldots, n$; and $1<p \leq q<\infty$, then for $0 \leq f$

$$
\left(\int_{\mathbb{R}_{+}^{n}} u(x)\left(\int_{0}^{x} f\right)^{q} d x\right)^{1 / q} \leq C\left(\int_{\mathbb{R}_{+}^{n}} f(x)^{p} v(x) d x\right)^{1 / p}
$$

is satisfied, if and only if $\max _{i=1, \ldots, n} A_{i} \equiv A<\infty$, where

$$
A_{i}=\sup _{s>0}\left(\int_{s}^{\infty} u_{i}(t) d t\right)^{1 / q}\left(\int_{0}^{s} v_{i}(t)^{1-p^{\prime}} d t\right)^{1 / p^{\prime}} .
$$

Similarly, if $0 \leq f$, then

$$
\left(\int_{\mathbb{R}_{+}^{n}} u(x)\left(\int_{x}^{\infty} f\right)^{q} d x\right)^{1 / q} \leq C\left(\int_{\mathbb{R}_{+}^{n}} f(x)^{p} v(x) d x\right)^{1 / p}
$$

holds, if and only if $\max _{i=1, \ldots, n} B_{i} \equiv B<\infty$, where

$$
B_{i}=\sup _{s>0}\left(\int_{0}^{s} u_{i}(t) d t\right)^{1 / q}\left(\int_{s}^{\infty} v_{i}(t)^{1-p^{\prime}} d t\right)^{1 / p^{\prime}}
$$

Moreover, if $C$ is the smallest constant for which (2.1), respectively (2.3) holds, then $A^{n} \leq C \leq\left[p^{1 / q}\left(p^{\prime}\right)^{1 / p^{\prime}} A\right]^{n}$, respectively, $B^{n} \leq C \leq$ $\left[p^{1 / q}\left(p^{\prime}\right)^{1 / p^{\prime}} B\right]^{n}$.

Proof The equivalence of (2.3) and (2.4) follows from the equivalence of (2.1) and (2.2) via a change of variables and is therefore omitted.

To prove $(2.1) \Rightarrow(2.2)$, define $r=\left(r_{1}, \ldots, r_{n}\right), r_{i}>0, i=1, \ldots, n$ and $f(x)=\prod_{i=1}^{n} v_{i}\left(x_{i}\right)^{1-p^{\prime}} \chi_{\left(0, r_{i}\right)}\left(x_{i}\right)$. For this $f$, we obtain from (2.1) that

$$
\begin{aligned}
& \prod_{i=1}^{n}\left(\int_{r_{i}}^{\infty} u_{i}\left(x_{i}\right) d x_{i}\right)^{1 / q}\left(\int_{0}^{r_{i}} v_{i}\left(t_{i}\right)^{1-p^{\prime}} d t_{i}\right) \\
& \quad \leq\left(\int_{\mathbb{R}_{+}^{n}} u(x)\left(\int_{0}^{x} f\right)^{q} d x\right)^{1 / q}
\end{aligned}
$$




$$
\begin{aligned}
& \leq C\left(\int_{\mathbb{R}_{+}^{n}} f(x)^{p} v(x) d x\right)^{1 / p} \\
& =C \prod_{i=1}^{n}\left(\int_{0}^{r_{i}} v_{i}\left(x_{i}\right)^{1-p^{\prime}} d x_{i}\right)^{1 / p} .
\end{aligned}
$$

Dividing by the product on the right hand it follows that

$$
\prod_{i=1}^{n}\left(\int_{r_{i}}^{\infty} u_{i}\right)^{1 / q}\left(\int_{0}^{r_{i}} v_{i}^{1-p^{\prime}}\right)^{1 / p^{\prime}} \leq C,
$$

so that $A^{n} \leq C$.

To prove the converse, we use induction: For $n=1$, the result is well known (cf. [7, Theorem 1.14]). If $\max _{i=1, \ldots, n-1} A_{i}<\infty$, and (2.1) is satisfied with $n$ replaced by $n-1$, then define

$$
F\left(x_{1}, \ldots, x_{n-1}, t_{n}\right)=\int_{0}^{x_{n-1}} \cdots \int_{0}^{x_{1}} f\left(t_{1}, \ldots, t_{n-1}, t_{n}\right) d t_{1} \cdots d t_{n-1} .
$$

From the one dimensional case, Minkowski's integral inequality and the induction assumption it follows that

$$
\begin{aligned}
&\left(\int_{\mathbb{R}_{+}^{n}} u(x)\left(\int_{0}^{x} f\right)^{q} d x\right)^{p / q} \\
&=\left\{\int_{\mathbb{R}_{+}^{n-1}} u_{1}\left(x_{1}\right) \cdots u_{n-1}\left(x_{n-1}\right)\right. \\
&\left.\quad \times\left[\int_{0}^{\infty} u_{n}\left(x_{n}\right)\left(\int_{0}^{x_{n}} F\left(x_{1}, \ldots, x_{n-1}, t_{n}\right) d t_{n}\right)^{q} d x_{n}\right] d x_{n-1} \cdots d x_{1}\right\}^{p / q} \\
& \leq\left[A_{n} p^{1 / q}\left(p^{\prime}\right)^{1 / p^{\prime}}\right]^{p}\left\{\int_{\mathbb{R}_{+}^{n-1}} u_{1}\left(x_{1}\right) \cdots u_{n-1}\left(x_{n-1}\right)\right. \\
&\left.\quad \times\left[\int_{0}^{\infty} v_{n}\left(t_{n}\right) F\left(x_{1}, \ldots, x_{n-1}, t_{n}\right)^{p} d t_{n}\right]^{q / p} d x_{n-1} \cdots d x_{1}\right\}^{p / q} \\
& \leq\left[A_{n} p^{1 / q}\left(p^{\prime}\right)^{1 / p^{\prime}}\right]^{p} \int_{0}^{\infty} v_{n}\left(t_{n}\right) \\
& \quad \times\left[\int_{\mathbb{R}_{+}^{n-1}} u_{1}\left(x_{1}\right) \cdots u_{n-1}\left(x_{n-1}\right) F\left(x_{1}, \ldots, x_{n-1}, t_{n}\right)^{q} d x_{n-1} \cdots d x_{1}\right]^{p / q} d t_{n} \\
& \leq {\left[A_{n} A_{n-1} \cdots A_{1}\left(p^{1 / q}\left(p^{\prime}\right)^{1 / p^{\prime}}\right)^{n}\right]^{p} \int_{\mathbb{R}_{+}^{n}} f(t)^{p} v(t) d t . }
\end{aligned}
$$


The first inequality holds, if and only if

$$
A_{n}=\sup _{s>0}\left(\int_{s}^{\infty} u_{n}\left(t_{n}\right) d t_{n}\right)^{1 / q}\left(\int_{0}^{s} v_{n}\left(t_{n}\right)^{1-p^{\prime}} d t_{n}\right)^{1 / p^{\prime}}<\infty .
$$

Hence the result follows.

We require the following special case of Theorem 2.1 in the next section:

Corollary 2.2 Suppose $v$ is a product weight on $\mathbb{R}_{+}^{n}$,

$$
V(x)=\int_{0}^{x} v, \quad V_{i}\left(x_{i}\right)=\int_{0}^{x_{i}} v_{i}, \quad i=1, \ldots, n
$$

and $1<p<\infty$. Then for $0 \leq f$

$$
\left(\int_{\mathbb{R}_{+}^{n}} v(x)\left(\int_{x}^{\infty} f\right)^{p} d x\right)^{1 / p} \leq C\left(\int_{\mathbb{R}_{+}^{n}} f(x)^{p} V(x)^{p} v(x)^{1-p} d x\right)^{1 / p}
$$

is satisfied. Moreover, the smallest $C$ in $(2.5)$ satisfies $\left(p^{\prime}-1\right)^{-n / p^{\prime}} \leq$ $C \leq p^{n}$.

Proof Apply (2.3) of Theorem 2.1 with $q=p, u$ replaced by $v$ and $v$ by $V^{p} v^{1-p}$. Then

$$
\begin{aligned}
B_{i} & =\sup _{s>0}\left(\int_{0}^{s} v_{i}(t) d t\right)^{1 / p}\left(\int_{s}^{\infty}\left[v_{i}(t)^{1-p} V_{i}(t)^{p}\right]^{1-p^{\prime}}\right)^{1 / p^{\prime}} \\
& =\sup _{s>0} V_{i}(s)^{1 / p}\left(\int_{s}^{\infty} v_{i}(t) V_{i}(t)^{-p^{\prime}} d t\right)^{1 / p^{\prime}} \leq\left(p^{\prime}-1\right)^{-1 / p^{\prime}}<\infty
\end{aligned}
$$

Next, we consider the following special case:

Corollary 2.3 Suppose $u(x)=u_{1}\left(x_{1}\right) u_{2}\left(x_{2}\right), v(x)=v_{1}\left(x_{1}\right) v_{2}\left(x_{2}\right), x \in$ $\mathbb{R}_{+}^{2}$ and $1<p \leq q<\infty$. Then for $0 \leq f$

$$
\left(\int_{\mathbb{R}_{+}^{2}} u(x)\left(\int_{0}^{x_{1}} \int_{x_{1}}^{\infty} f\right)^{q} d x\right)^{1 / q} \leq C\left(\int_{\mathbb{R}_{+}^{2}} f^{p}(x) v(x) d x\right)^{1 / p}
$$


is satisfied if and only if

$$
\begin{aligned}
& \sup _{s>0}\left(\int_{s}^{\infty} u_{1}\right)^{1 / q}\left(\int_{0}^{s} v_{1}^{1-p^{\prime}}\right)^{1 / p^{\prime}}<\infty ; \\
& \sup _{s>0}\left(\int_{s}^{\infty} u_{2}\right)^{1 / q}\left(\int_{0}^{s} v_{2}^{1-p^{\prime}}\right)^{1 / p^{\prime}}<\infty .
\end{aligned}
$$

Proof Let $\tilde{f}\left(t_{1}, t_{2}\right)=f\left(t_{1}, 1 / t_{2}\right) / t_{2}^{2}, \quad \tilde{u}\left(x_{1}, x_{2}\right)=u\left(x_{1}, 1 / x_{2}\right) / x_{2}^{2}$, $\tilde{v}\left(t_{1}, t_{2}\right)=t_{2}^{-2 p-2} v\left(t_{1}, 1 / t_{2}\right)$, then the left side of $(2.6)$ is

$$
\begin{aligned}
\left(\int_{\mathbb{R}_{+}^{2}} \tilde{u}(x)\left(\int_{0}^{x} \tilde{f}\right)^{q}\right)^{1 / q} & \leq C\left(\int_{\mathbb{R}_{+}^{2}} \tilde{v}(t) \tilde{f}(t)^{p} d t\right)^{1 / p} \\
& =C\left(\int_{\mathbb{R}_{+}^{2}} f(x)^{p} v(x) d x\right)^{1 / p}
\end{aligned}
$$

by Theorem 2.1, if and only if the first supremum of (2.7) holds and

$$
\sup _{s>0}\left(\int_{s}^{\infty} \frac{u_{2}(1 / t)}{t^{2}} d t\right)^{1 / q}\left(\int_{0}^{s} t^{-2(p+1)\left(1-p^{\prime}\right)} v_{2}(1 / t)^{1-p^{\prime}} d t\right)^{1 / p^{\prime}}<\infty .
$$

But the obvious change of variable shows that this is equivalent to the second supremum of (2.7).

It is clear that a higher dimensional analogue of Corollary 2.3 may be proved in the same way.

The following standard duality theorem for $L^{p_{-}}$and $\ell^{p}$-spaces is stated for completeness only.

Theorem 2.4

(a) If $(X, \nu)$ is a measure space and $1<p<\infty$, then for $0 \leq g$

$$
\left(\int_{X} g^{p^{\prime}} d \nu\right)^{1 / p^{\prime}}=\sup _{0 \leq f} \frac{\int_{X} f g d \nu}{\left(\int_{X} f^{p} d \nu\right)^{1 / p}}
$$

(b) If $v$ is a weight function, then

$$
\left(\int_{X} g^{p^{\prime}} v^{1-p^{\prime}}\right)^{1 / p^{\prime}}=\sup _{0 \leq f} \frac{\int_{X} f g}{\left(\int_{X} f^{p} v\right)^{1 / p}} .
$$


(c) The corresponding discrete analogues for (2.8) and (2.9) hold also and constitute duality theorems in $\ell^{p}$-spaces.

The discrete analogue of Theorem 2.1 follows from the following result proved by Sinnamon [9] and Bennett [4]:

Theorem $2.5[9,4]$ Suppose $\left\{u_{n}\right\}_{n=1}^{\infty},\left\{v_{n}\right\}_{n=1}^{\infty}$ are strictly positive (weight) sequences and $1<p \leq q<\infty$. If $a \equiv\left\{a_{n}\right\}_{n=1}^{\infty}$ is a positive sequence, then the Hardy-Césaro inequality

$$
\left[\sum_{n=1}^{\infty} u_{n}\left(\sum_{k=1}^{n} a_{k}\right)^{q}\right]^{1 / q} \leq C\left[\sum_{n=1}^{\infty} a_{n}^{p} v_{n}\right]^{1 / p}
$$

is satisfied, if and only if

$$
\sup _{m>1}\left(\sum_{n=m}^{\infty} u_{n}\right)^{1 / q}\left(\sum_{n=1}^{m} v_{n}^{1-p^{\prime}}\right)^{1 / p^{\prime}}<\infty .
$$

For the conjugate Copson operator, the inequality

$$
\left[\sum_{n=1}^{\infty} u_{n}\left(\sum_{k=n}^{\infty} a_{k}\right)^{q}\right]^{1 / q} \leq C\left[\sum_{n=1}^{\infty} a_{n}^{p} v_{n}\right]^{1 / p}
$$

is satisfied, if and only if

$$
\sup _{m>0}\left(\sum_{n=1}^{m} u_{n}\right)^{1 / q}\left(\sum_{n=m}^{\infty} v_{n}^{1-p^{\prime}}\right)^{1 / p^{\prime}}<\infty .
$$

We also require a discrete analogue of Corollary 2.2 for the case $n=2$.

Corollary 2.6 Suppose $\left\{v_{i, j}\right\}_{i, j=1}^{\infty}$ is a product weight sequence: $v_{i, j}=$ $v_{i}^{\prime} v_{j}^{\prime \prime}$ and $V_{i}^{\prime}=\sum_{k=1}^{i} v_{k}^{\prime}, V_{j}^{\prime \prime}=\sum_{k=1}^{j} v_{k}^{\prime \prime}$ and $1<p<\infty$. If $a=\left\{a_{i, j}\right\}_{i, j=1}^{\infty}$ is a positive sequence, then

$$
\sum_{i, j=1}^{\infty} v_{i, j}\left(\sum_{n=i}^{\infty} \sum_{m=j}^{\infty} a_{n, m}\right)^{p} \leq C \sum_{i, j=1}^{\infty} a_{i, j}^{p}\left(V_{i}^{\prime} V_{j}^{\prime \prime}\right)^{p} v_{i, j}^{1-p}
$$

is satisfied. 
Proof Define $\left\{b_{n, j}\right\}$ by $b_{n, j}=\sum_{m=j}^{\infty} a_{n, m}$, then the left side of (2.10) becomes

$$
\sum_{j=1}^{\infty} v_{j}^{\prime \prime}\left[\sum_{i=1}^{\infty} v_{i}^{\prime}\left(\sum_{n=i}^{\infty} b_{n, j}\right)^{p}\right] \leq C^{p} \sum_{j=1}^{\infty} v_{j}^{\prime \prime}\left[\sum_{i=1}^{\infty} b_{i, j}^{p}\left(V_{i}^{\prime}\right)^{p}\left(v_{i}^{\prime}\right)^{1-p}\right]
$$

by Theorem 2.5 with $q=p$, provided that for each $m \in \mathbb{N}$

$$
\left(\sum_{i=1}^{m} v_{i}^{\prime}\right)^{1 / p}\left(\sum_{i=m}^{\infty}\left[\left(V_{i}^{\prime}\right)^{p}\left(v_{i}^{\prime}\right)^{1-p}\right]^{1-p^{\prime}}\right)^{1 / p^{\prime}}
$$

is bounded. But using the fact that for any positive sequence $\left\{c_{n}\right\}_{n=1}^{\infty}$ and $\alpha>1$

$$
\sum_{n=m}^{\infty} c_{n}\left(\sum_{k=1}^{n} c_{k}\right)^{-\alpha} \leq \alpha^{\prime}\left(\sum_{k=1}^{m} c_{k}\right)^{1-\alpha}
$$

holds for $m \in \mathbb{N}$, the second product of (2.12) becomes

$$
\left(\sum_{i=m}^{\infty}\left(V_{i}^{\prime}\right)^{-p^{\prime}} v_{i}^{\prime}\right)^{1 / p^{\prime}} \leq p\left(\sum_{i=1}^{m} v_{i}\right)^{\left(1-p^{\prime}\right) / p^{\prime}}=p\left(\sum_{i=1}^{m} v_{i}\right)^{-1 / p} .
$$

Hence (2.12) is bounded by $p$ and thus, (2.11) is satisfied. Since the sum on the right side of (2.11) is

$$
\begin{aligned}
& \sum_{i=1}^{\infty}\left(V_{i}^{\prime}\right)^{p}\left(v_{i}^{\prime}\right)^{1-p} \sum_{j=1}^{\infty} v_{j}^{\prime \prime}\left(\sum_{m=j}^{\infty} a_{i, m}\right)^{p} \\
& \quad \leq C^{p} \sum_{i=1}^{\infty}\left(V_{i}^{\prime}\right)^{p}\left(v_{i}^{\prime}\right)^{1-p} \sum_{j=1}^{\infty} a_{i, j}^{p}\left(V_{j}^{\prime \prime}\right)^{p}\left(v_{j}^{\prime \prime}\right)^{1-p} \\
& \quad=C^{p} \sum_{i, j=1}^{\infty}\left(V_{i}^{\prime} V_{j}^{\prime \prime}\right)^{p} v_{i, j}^{1-p} a_{i, j}^{p}
\end{aligned}
$$

by Theorem 2.5 with $q=p$, whenever (2.12) holds with $v_{i}^{\prime}$ replaced by $v_{j}^{\prime \prime}$, the results follows.

Remark 2.7 In a similar way one can obtain weighted inequalities of the form (2.10) where the double sum on the left of (2.10) is replaced by $\sum_{n=1}^{i} \sum_{m=1}^{j}$ or $\sum_{n=1}^{i} \sum_{m=j}^{\infty}$. 
We conclude this section by recalling the following consequence of Abel's summation formula: If $\left\{b_{k}\right\}_{k=1}^{\infty},\left\{v_{k}\right\}_{k=1}^{\infty}$ are positive sequences,

$$
B_{n}=\sum_{k=1}^{n} b_{k}, \quad n \geq 1, B_{0}=0 ; \quad V_{n}=\sum_{k=1}^{n} v_{k}, \quad n \in \mathbb{N},
$$

then

$$
\sum_{n=k}^{\infty} b_{n} V_{n}^{-1} \leq V_{\infty}^{-1} B_{\infty}+\sum_{n=k}^{\infty} B_{n} v_{n+1} V_{n}^{-1} V_{n+1}^{-1}
$$

\section{DUALITY THEOREMS FOR FUNCTIONS}

We first consider the case for functions of two variables, decreasing in each variable separately. The general case - as we shall see - follows along the same lines.

THEOREM 3.1 Suppose $v$ is a product weight on $\mathbb{R}_{+}^{2}, V_{i}\left(x_{i}\right)=\int_{0}^{x_{i}} v_{i}(t) d t$, $i=1,2$ and $1<p<\infty$. If $g \geq 0$, then

$$
\sup _{0 \leq f \Downarrow} \frac{\int_{\mathbb{R}_{+}^{2}} f g}{\left(\int_{\mathbb{R}_{+}^{2}} f^{p} v\right)^{1 / p}} \approx \sum_{k=1}^{4} I_{k},
$$

where

$$
\begin{gathered}
I_{1}=V(\infty)^{-1 / p}\|g\|_{1}, \\
I_{2}=V_{2}(\infty)^{-1 / p}\left\{\int_{0}^{\infty}\left(\int_{0}^{x_{1}}\|g(s, \cdot)\|_{1} d s\right)^{p^{\prime}}\right. \\
\left.V_{1}^{-p^{\prime}}\left(x_{1}\right) v_{1}\left(x_{1}\right) d x_{1}\right\}^{1 / p^{\prime}}, \\
I_{3}=V_{1}(\infty)^{-1 / p}\left\{\int_{0}^{\infty}\left(\int_{0}^{x_{2}}\|g(\cdot, t)\|_{1} d t\right)^{p^{\prime}}\right. \\
I_{4}=\left\{\int_{\mathbb{R}_{+}^{2}}\left(\int_{0}^{x} g\right)^{p^{\prime}} V(x)^{-p^{\prime}} v(x) d x\right\}^{1 / p^{\prime}},
\end{gathered}
$$

where $V(x)=V_{1}\left(x_{1}\right) V_{2}\left(x_{2}\right)$. 
Proof The argument of the proof follows along the lines of the one dimensional theorem proved by Stepanov [10].

Let $O(g)$ denote the left hand side of (3.1), then for the proof of the lower bound we consider several cases:

If $f=C>0$, a constant, then

$$
O(g) \geq \sup _{f=c} \frac{\int_{\mathbb{R}_{+}^{2}} f g}{\left(\int_{\mathbb{R}_{+}^{2}} f^{p} v\right)^{1 / p}}=\frac{\|g\|_{1}}{V(\infty)^{1 / p}}=I_{1} .
$$

If $f\left(x_{1}, x_{2}\right)$ is decreasing in $x_{1}$ only, let $f\left(x_{1}, x_{2}\right)=\int_{x_{1}}^{\infty} h$, where $h$ on $\mathbb{R}_{+}$ is arbitrary. Then

$$
\begin{aligned}
O(g) & \geq \sup _{f(x)=\int_{x_{1}}^{\infty} h} \frac{\int_{\mathbb{R}_{+}^{2}} f g}{\left(\int_{\mathbb{R}_{+}^{2}} f^{p} v\right)^{1 / p}}=\sup _{h \geq 0} \frac{\int_{\mathbb{R}_{+}^{2}} g(x)\left(\int_{x_{1}}^{\infty} h\right) d x}{\left(\int_{\mathbb{R}_{+}^{2}} v(x)\left(\int_{x_{1}}^{\infty} h\right)^{p} d x\right)^{1 / p}} \\
& =\sup _{h \geq 0} \frac{\int_{0}^{\infty} h\left(t_{1}\right)\left(\int_{0}^{t_{1}}\left\|g\left(x_{1}, \cdot\right)\right\|_{1} d x_{1}\right) d t_{1}}{\left\{\int_{0}^{\infty} v_{2}\left(x_{2}\right)\left[\int_{0}^{\infty} v_{1}\left(x_{1}\right)\left(\int_{x_{1}}^{\infty} h\right)^{p} d x_{1}\right] d x_{2}\right\}^{1 / p}} .
\end{aligned}
$$

Applying Corollary 2.2 with $n=1$ the inner integral of the denominator is dominated by

$$
\int_{0}^{\infty} h\left(x_{1}\right)^{p} V_{1}\left(x_{1}\right)^{p} v_{1}\left(x_{1}\right)^{1-p} d x_{1}
$$

so that

$$
\begin{aligned}
O(g) & \geq p^{-1} V_{2}(\infty)^{-1 / p} \sup _{h \geq 0} \frac{\int_{0}^{\infty}\left(h V_{1} / v_{1}\right)\left(v_{1} / V_{1}\right)\left(\int_{0}^{t_{1}}\left\|g\left(x_{1}, \cdot\right)\right\|_{1} d x_{1}\right) d t_{1}}{\left(\int_{0}^{\infty}\left(h V_{1} / v_{1}\right)^{p} v_{1}\right)^{1 / p}} \\
& =p^{-1} V_{2}(\infty)^{-1 / p}\left(\int_{0}^{\infty}\left(\int_{0}^{t_{1}}\left\|g\left(x_{1}, \cdot\right)\right\|_{1} d x_{1}\right)^{p^{\prime}} V_{1}\left(t_{1}\right)^{-p^{\prime}} v_{1}\left(t_{1}\right) d t_{1}\right)^{1 / p^{\prime}} \\
& =p^{-1} I_{2}
\end{aligned}
$$

where the last inequality follows from Theorem 2.4 (a) with $X=\mathbb{R}_{+}, d v(x)=v_{1}\left(x_{1}\right) d x_{1}, f$ replaced by $\left(h V_{1} / v\right)$ and $g$ by $V_{1}\left(t_{1}\right)^{-1}\left(\int_{0}^{t_{1}}\left\|g\left(x_{1}, \cdot\right)\right\|_{1} d x_{1}\right)$.

If $f\left(x_{1}, x_{2}\right)$ is decreasing in $x_{2}$ only, the argument is completely analogous and we obtain $O(g) \geq p^{-1} I_{3}$.

If $f$ is decreasing in both variables, let $f(x)=\int_{x}^{\infty} h$, where $h$ on $\mathbb{R}_{+}^{2}$ is arbitrary. If $G(t)=\int_{0}^{t} g, t=\left(t_{1}, t_{2}\right)$, then an interchange of order of 
integration and Corollary 2.2 with $n=2$ show that

$$
\begin{aligned}
O(h) & \geq \sup _{h \geq 0} \frac{\int_{\mathbb{R}_{+}^{2}} g(x)\left(\int_{x}^{\infty} h\right) d x}{\left(\int_{\mathbb{R}_{+}^{2}} v(x)\left(\int_{x}^{\infty} h\right)^{p} d x\right)^{1 / p}} \geq p^{-2} \sup _{h \geq 0} \frac{\int_{\mathbb{R}_{+}^{2}} h G}{\left(\int_{\mathbb{R}_{+}^{2}} h^{p} V^{p} v^{1-p}\right)^{1 / p}} \\
& =p^{-2} \sup _{h \geq 0} \frac{\int_{\mathbb{R}_{+}^{2}}(h V / v)(v / V) G}{\left(\int_{\mathbb{R}_{+}^{2}}(h V / v)^{p} v\right)^{1 / p}}=p^{-2}\left(\int_{\mathbb{R}_{+}^{2}}\left(\frac{G}{V}\right)^{p^{\prime}} v\right)^{1 / p^{\prime}} \\
& =p^{-2} I_{4}
\end{aligned}
$$

where again Theorem 2.4 has been applied. Clearly the lower bound follows from these estimates.

To prove the upper bound of $O(g)$ we interchange the order of integration several times and use the monotonicity of $f$ in both its variables to obtain

$$
\begin{aligned}
\int_{\mathbb{R}_{+}^{2}} g f= & \int_{\mathbb{R}_{+}^{2}} g f V^{-1} V \\
= & \int_{0}^{\infty} V_{1}\left(x_{1}\right) V_{1}\left(x_{1}\right)^{-1} \\
& {\left[\int_{0}^{\infty} g(x) f(x) V_{2}\left(x_{2}\right)^{-1}\left(\int_{0}^{x_{2}} v_{2}\left(t_{2}\right) d t_{2}\right) d x_{2}\right] d x_{1} } \\
= & \int_{0}^{\infty} V_{1}\left(x_{1}\right) V_{1}^{-1}\left(x_{2}\right) \int_{0}^{\infty} v_{2}\left(t_{2}\right) \\
& {\left[\int_{t_{2}}^{\infty} g(x) f(x) V_{2}^{-1}\left(x_{2}\right) d x_{2}\right] d t_{2} d x_{1} } \\
\leq & \int_{0}^{\infty} V_{1}\left(x_{1}\right) V_{1}\left(x_{1}\right)^{-1} \int_{0}^{\infty} v_{2}\left(t_{2}\right) f\left(x_{1}, t_{2}\right) \\
= & \int_{0}^{\infty} v_{2}\left(t_{2}\right) \int_{0}^{\infty} f\left(x_{1}, t_{2}\right) V_{1}\left(x_{1}\right)^{-1}\left(\int_{t_{2}}^{\infty} g(x) V_{2}\left(x_{2}\right)^{-1} d x_{2}\right) \\
& \left.\left.\times\left(\int_{0}^{x_{1}} v_{1}\left(t_{1}\right) d t_{1}\right) d x_{1} d t_{1}\right) d x_{2}\right) d t_{2} d x_{1}
\end{aligned}
$$




$$
\begin{aligned}
= & \int_{0}^{\infty} v_{2}\left(t_{2}\right) \int_{0}^{\infty} v_{1}\left(t_{1}\right) \int_{t_{1}}^{\infty} f\left(x_{1}, t_{2}\right) V_{1}\left(x_{1}\right)^{-1} \\
& \times\left(\int_{t_{2}}^{\infty} g(x) V_{2}\left(x_{2}\right)^{-1} d x_{2}\right) d x_{1} d t_{1} d t_{2} \\
\leq & \int_{\mathbb{R}_{+}^{2}} v(t) f(t)\left(\int_{t}^{\infty} g(x) V(x)^{-1} d x\right) d t .
\end{aligned}
$$

To estimate the inner integral of (3.2), observe first that

$$
\begin{aligned}
\int_{t_{2}}^{\infty} g(x) V_{2}\left(x_{2}\right)^{-1} d x_{2} \\
=\int_{t_{2}}^{\infty} V_{2}\left(x_{2}\right)^{-1} d\left(\int_{0}^{x_{2}} g\left(x_{1}, s_{2}\right) d s_{2}\right) \\
=\left.V_{2}\left(x_{2}\right)^{-1} \int_{0}^{x_{2}} g\left(x_{1}, s_{2}\right) d s_{2}\right|_{t_{2}} ^{\infty} \\
\quad+\int_{t_{2}}^{\infty} V_{2}\left(x_{2}\right)^{-2} v_{2}\left(x_{2}\right)\left(\int_{0}^{x_{2}} g\left(x_{1}, s_{2}\right) d s_{2}\right) d x_{2} \\
\leq V_{2}(\infty)^{-1}\left\|g\left(x_{1}, \cdot\right)\right\|_{1}+\int_{t_{2}}^{\infty} V_{2}\left(x_{2}\right)^{-2} v_{2}\left(x_{2}\right) \\
\quad\left(\int_{0}^{x_{2}} g\left(x_{1}, s_{2}\right) d s_{2}\right) d x_{2} .
\end{aligned}
$$

Therefore

$$
\begin{aligned}
\int_{t}^{\infty} & g(x) V(x)^{-1} d x \\
\leq & \int_{t_{1}}^{\infty} V_{1}\left(x_{1}\right)^{-1}\left[V_{2}(\infty)^{-1}\left\|g\left(x_{1}, \cdot\right)\right\|_{1}\right. \\
& \left.\quad+\int_{t_{2}}^{\infty} V_{2}\left(x_{2}\right)^{-1} v_{2}\left(x_{2}\right)\left(\int_{0}^{x_{2}} g\left(x_{1}, s_{2}\right) d s_{2}\right) d x_{2}\right] d x_{1} \\
= & V_{2}(\infty)^{-1} \int_{t_{1}}^{\infty} V_{1}\left(x_{1}\right)^{-1}\left\|g\left(x_{1}, \cdot\right)\right\|_{1} d x_{1} \\
& +\int_{t_{2}}^{\infty} V_{2}\left(x_{2}\right)^{-2} v_{2}\left(x_{2}\right) \int_{t_{1}}^{\infty} V_{1}\left(x_{1}\right)^{-1}\left(\int_{0}^{x_{2}} g\left(x_{1}, s_{2}\right) d s_{2}\right) d x_{1} d x_{2} \\
\equiv & J_{1}+J_{2},
\end{aligned}
$$


respectively. Again integrating by parts shows that

$$
\begin{aligned}
& J_{1}= V_{2}(\infty)^{-1} \int_{t_{1}}^{\infty} V_{1}\left(x_{1}\right)^{-1} d\left(\int_{0}^{x_{1}}\left\|g\left(s_{1}, \cdot\right)\right\| d s_{1}\right) \\
& \leq V_{2}(\infty)^{-1}\left[V_{1}(\infty)^{-1}\|g\|_{1}+\int_{t_{1}}^{\infty} V_{1}\left(x_{1}\right)^{-2} v_{1}\left(x_{1}\right)\right. \\
&\left.\quad\left(\int_{0}^{x_{1}}\left\|g\left(s_{1}, \cdot\right)\right\|_{1} d s_{1}\right) d x_{1}\right] \equiv K_{1}+K_{2}, \\
& J_{2}=\int_{t_{2}}^{\infty} V_{2}\left(x_{2}\right)^{-2} v_{2}\left(x_{2}\right)\left[\int_{t_{1}}^{\infty} V_{1}\left(x_{1}\right)^{-1} d\right. \\
&\left.\quad\left(\int_{0}^{x_{1}}\left(\int_{0}^{x_{2}} g\left(s_{1}, s_{2}\right) d s_{2}\right) d s_{1}\right)\right] d x_{2} \\
&=\int_{t_{2}}^{\infty} V_{2}\left(x_{2}\right)^{-2} v_{2}\left(x_{2}\right)\left[\left.V_{1}\left(x_{1}\right)^{-1}\left(\int_{0}^{x} g\right)\right|_{t_{1}} ^{\infty} V_{1}\left(x_{1}\right)^{-2} v_{1}\left(x_{1}\right)\left(\int_{0}^{x} g\right) d x_{1}\right] d x_{2} \\
& \leq V_{1}(\infty)^{-1} \int_{t_{2}}^{\infty} V_{2}\left(x_{2}\right)^{-2} v_{2}\left(x_{2}\right)\left(\int_{0}^{x_{2}}\left\|g\left(\cdot, s_{2}\right)\right\|_{1} d s_{2}\right) d x_{2} \\
&+\int_{t}^{\infty} V(x)^{-2} v(x)\left(\int_{0}^{x} g\right) d x \equiv K_{3}+K_{4},
\end{aligned}
$$

respectively. Hence the inner integral of (3.2) is estimated by $\sum_{j=1}^{4} K_{j}$. Substituting and applying Hölder's and Minkowski's inequalities we obtain

$$
\begin{aligned}
\int_{\mathbb{R}_{+}^{2}} g f & \leq \int_{\mathbb{R}_{+}^{2}} f v\left(\sum_{j=1}^{4} K_{j}\right) \\
& \leq\left(\int_{\mathbb{R}_{+}^{2}} f^{p} v\right)^{1 / p}\left(\int_{\mathbb{R}_{+}^{2}} v\left[\sum_{j=1}^{4} K_{j}\right]^{p^{\prime}}\right)^{1 / p^{\prime}} \\
& \leq\left(\int_{\mathbb{R}_{+}^{2}} f^{p} v\right)^{1 / p}\left(\sum_{j=1}^{4}\left(\int_{\mathbb{R}_{+}^{2}} v K_{j}^{p^{\prime}}\right)^{1 / p^{\prime}}\right) .
\end{aligned}
$$

Now

$$
\begin{aligned}
\left(\int_{\mathbb{R}_{+}^{2}} v K_{1}^{p^{\prime}}\right)^{1 / p^{\prime}} & =V(\infty)^{-1}\|g\|_{1}\left(\int_{\mathbb{R}_{+}^{2}} v\right)^{1 / p^{\prime}} \\
& =V(\infty)^{-1 / p^{\prime}}\|g\|_{1}=I_{1} .
\end{aligned}
$$


According to Corollary 2.2 with $n=1$ we have

$$
\begin{aligned}
& \left(\int_{\mathbb{R}_{+}^{2}} v K_{2}^{p^{\prime}}\right)^{1 / p^{\prime}} \\
& =V_{2}(\infty)^{-1}\left\{\int _ { 0 } ^ { \infty } v _ { 2 } ( t _ { 2 } ) \left[\int _ { 0 } ^ { \infty } v _ { 1 } ( t _ { 1 } ) \left(\int_{t_{1}}^{\infty} V_{1}^{-2}\left(x_{1}\right) v_{1}\left(x_{1}\right)\right.\right.\right. \\
& \left.\left.\left.\quad \times\left(\int_{0}^{x_{1}}\left\|g\left(s_{1}, \cdot\right)\right\| d s_{1}\right) d x_{1}\right)^{p^{\prime}} d t_{1}\right] d t_{2}\right\}^{1 / p^{\prime}} \\
& \leq p^{\prime} V_{2}(\infty)^{-1 / p}\left[\int_{0}^{\infty} v_{1}\left(t_{1}\right)^{1-p^{\prime}} V_{1}^{p^{\prime}}\left(t_{1}\right) V_{1}^{-2 p^{\prime}}\left(t_{1}\right) v_{1}\left(t_{1}\right)^{p^{\prime}}\right. \\
& \left.\times\left(\int_{0}^{t_{1}}\left\|f\left(s_{1}, \cdot\right)\right\|_{1} d s_{1}\right)^{p^{\prime}} d t_{1}\right]^{1 / p^{\prime}} \\
& =p^{\prime} V_{2}(\infty)^{-1 / p}\left[\int_{0}^{\infty} V_{1}\left(t_{1}\right)^{-p^{\prime}}\left(\int_{0}^{t_{1}}\left\|g\left(s_{1}, \cdot\right)\right\|_{1} d s_{1}\right)^{p^{\prime}} v_{1}\left(t_{1}\right) d t_{1}\right]^{1 / p^{\prime}} \\
& =p^{\prime} I_{2} .
\end{aligned}
$$

By symmetry the same argument applies to yield

$$
\left(\int_{\mathbb{R}_{+}^{2}} v K_{3}^{p^{\prime}}\right)^{1 / p^{\prime}} \leq p^{\prime} I_{3} .
$$

Finally, by Corollary 2.2 with $n=2, p$ replaced by $p^{\prime}$ and $f$ by $V^{-2} v \int_{0}^{x} g$ we find

$$
\begin{aligned}
\left(\int_{\mathbb{R}_{+}^{2}} v K_{4}^{p^{\prime}}\right)^{1 / p^{\prime}} & =\left(\int _ { \mathbb { R } _ { + } ^ { 2 } } v ( t ) \left(\int_{t}^{\infty} V(x)^{-2} v(x)\right.\right. \\
& \left.\left.\left(\int_{0}^{x} g\right) d x\right)^{p^{\prime}} d t\right)^{1 / p^{\prime}} \\
& \leq\left(p^{\prime}\right)^{2}\left(\int_{\mathbb{R}_{+}^{2}} V(x)^{-p^{\prime}}\left(\int_{0}^{x} g\right)^{p^{\prime}} v(x) d x\right)^{1 / p^{\prime}} \\
& =\left(p^{\prime}\right)^{2} I_{4} .
\end{aligned}
$$

Dividing by the first integral factor we obtain

$$
\frac{\int_{\mathbb{R}_{+}^{2}} f g}{\left(\int_{\mathbb{R}_{+}^{2}} f^{p} v\right)^{1 / p}} \leq I_{1}+p^{\prime}\left(I_{1}+I_{2}\right)+\left(p^{\prime}\right) I_{4}
$$

from which the upper bound of $O(g)$ follows. 
It is clear that if $V_{i}(\infty)=\infty, i=1,2$, then $I_{1}=I_{2}=I_{3}=0$. Assuming these conditions on the product weights we obtain

COROLlaRY 3.2 Suppose $v$ is a product weight and $V_{i}\left(x_{i}\right)=\int_{0}^{x_{i}} v_{i}$ satisfies $V_{i}(\infty)=\infty, i=1, \ldots, n$. If $1<p<\infty$, then

$$
\sup _{0 \leq f \Downarrow} \frac{\int_{\mathbb{R}_{+}^{n}} f g}{\left(\int_{\mathbb{R}_{+}^{n}} f^{p} v\right)^{1 / p}} \approx\left(\int_{\mathbb{R}_{+}^{n}}\left(\int_{0}^{x} g\right)^{p^{\prime}} V(x)^{-p^{\prime}} v(x) d x\right)^{1 / p^{\prime}} .
$$

Proof It is clear that all terms for the upper and lower bound are zero except one. The non-zero term for the lower bound arises when $f$ is taken as $f(x)=\int_{x}^{\infty} h, h$ on $\mathbb{R}_{+}^{n}$ and for the upper bound the only nonzero term is

$$
\left(\int_{\mathbb{R}_{+}^{n}} v\left(\int_{t}^{\infty} V(x)^{-2} v(x)\left(\int_{0}^{x} g\right) d x\right)^{p^{\prime}} d t\right)^{1 / p^{\prime}} .
$$

In both cases we apply Corollary 2.2 to obtain the result.

Corollary 3.3 Suppose $1<p<\infty, g \geq 0$ and $v$ a product weight such that $V_{i}^{*}\left(x_{i}\right)=\int_{x_{i}}^{\infty} v_{i}$ satisfies $V_{i}^{*}(0)=\infty, i=1, \ldots, n$. Then

$$
\sup _{0 \leq f \Uparrow} \frac{\int_{\mathbb{R}_{+}^{n}} f g}{\left(\int_{\mathbb{R}_{+}^{n}} f^{p} v\right)^{1 / p}} \approx\left(\int_{\mathbb{R}_{+}^{n}}\left(\int_{x}^{\infty} g\right)^{p^{\prime}} V^{*}(x)^{-p^{\prime}} v(x) d x\right)^{1 / p^{\prime}} .
$$

Proof If $0 \leq f \Uparrow$ then $\tilde{f}(x)=f(1 / x) \Downarrow$. If $\tilde{g}(x)=g(1 / x) / x^{2}, \tilde{v}(x)=$ $v(1 / x) / x^{2}$ then by (3.4) with $f$ replaced by $\tilde{f}, g$ by $\tilde{g}, v$ by $\tilde{v}$ where $\tilde{V}(x)=\int_{0}^{x} \tilde{v}$ we obtain

$$
\begin{aligned}
\sup _{0 \leq f \Uparrow} \frac{\int_{\mathbb{R}_{+}^{n}} f g}{\left(\int_{\mathbb{R}_{+}^{n}} f^{p} v\right)^{1 / p}} & =\sup _{0 \leq f \Downarrow} \frac{\int_{\mathbb{R}_{+}^{n}} \tilde{f} \tilde{g}}{\left(\int_{\mathbb{R}_{+}^{n}} \tilde{f}^{p} \tilde{v}\right)^{1 / p}} \\
& \approx\left(\int_{\mathbb{R}_{+}^{n}}\left(\int_{0}^{x} \tilde{g}\right)^{p^{\prime}} \tilde{V}^{-p^{\prime}}(x) \tilde{v}(x) d x\right)^{1 / p^{\prime}} \\
& =\left(\int_{\mathbb{R}_{+}^{n}}\left(\int_{1 / x}^{\infty} g\right)^{p^{\prime}}\left(\int_{1 / x}^{\infty} v\right)^{p^{\prime}} \tilde{v}(x) d x\right)^{1 / p^{\prime}} \\
& =\left(\int_{\mathbb{R}_{+}^{n}}\left(\int_{x}^{\infty} g\right)^{p^{\prime}} V^{*}(x)^{-p^{\prime}} v(x) d x\right)^{1 / p^{\prime}} .
\end{aligned}
$$

Note that $V^{*}(0)=\infty$ if and only if $\widetilde{V}(\infty)=\infty$. 
It is clear from this result how a duality theorem of a function $f$ of mixed monotonicity is obtained. For example if $f\left(x_{1}, x_{2}\right)$ is decreasing in $x_{1}$ and increasing in $x_{2}$ (write $0 \leq f \uparrow \downarrow$ ) we obtain

Corollary 3.4 Suppose $1<p<\infty, g \geq 0$, v a product weight on $\mathbb{R}_{+}^{2}$, where $V_{1}\left(x_{1}\right)=\int_{0}^{x_{1}} v_{1}, V_{2}^{*}\left(x_{2}\right)=\int_{x_{2}}^{\infty} v_{2}$ satisfies $V_{1}(\infty)=V_{2}^{*}(0)=\infty$. Then

$$
\begin{aligned}
\sup _{0 \leq f \downarrow \uparrow} \frac{\int_{\mathbb{R}_{+}^{2}} f g}{\left(\int_{\mathbb{R}_{+}^{2}} f^{p} v\right)^{1 / p}} \\
\approx\left(\int_{\mathbb{R}_{+}^{2}}\left(\int_{0}^{x_{1}} \int_{x_{2}}^{\infty} g\right)^{p^{\prime}} V_{1}\left(x_{1}\right)^{-p^{\prime}} V_{2}^{*}\left(x_{2}\right)^{-p^{\prime}} v(x) d x\right)^{1 / p^{\prime}} .
\end{aligned}
$$

Proof Define $\tilde{f}\left(x_{1}, x_{2}\right)=f\left(x_{1}, 1 / x_{2}\right), \quad \tilde{g}\left(x_{1}, x_{2}\right)=g\left(x_{1}, 1 / x_{2}\right) / x_{2}$, $\tilde{v}_{2}\left(x_{2}\right)=v_{2}\left(x_{2}\right) / x_{2}, \tilde{V}_{2}\left(x_{2}\right)=\int_{0}^{x_{1}} \tilde{v}_{2}$. Then from obvious changes of variables Corollary 3.2 applies and the result follows.

\section{A DUALITY THEOREM FOR MONOTONE SEQUENCES}

In this section we prove the discrete analogue of Theorem 3.1 under the condition that the sums of the product weight series diverge.

THEOREM 4.1 Suppose $\left\{v_{i, j}\right\}_{i, j=1}^{\infty}$ is a product weight sequence such that $V_{n}^{\prime}=\sum_{i=1}^{n} v_{i}^{\prime}, V_{n}^{\prime \prime}=\sum_{j=1}^{n} v_{j}^{\prime \prime}, v_{i, j}=v_{i}^{\prime} v_{j}^{\prime \prime}$ satisfies $V_{\infty}^{\prime}=V_{\infty}^{\prime \prime}=\infty$. If $1<$ $p<\infty$ and $b=\left\{b_{i, j}\right\}_{i, j=1}^{\infty} \geq 0$, then

$$
\begin{aligned}
& \sup _{0 \leq a \Downarrow} \frac{\sum_{i, j=1}^{\infty} a_{i, j} b_{i, j}}{\left(\sum_{i, j=1}^{\infty} a_{i, j}^{p} v_{i, j}\right)^{1 / p}} \\
& \approx\left(\sum_{i, j=1}^{\infty}\left[\frac{1}{V_{i}^{\prime} V_{j}^{\prime}} \sum_{k=1}^{i} \sum_{n=1}^{j} b_{k, n}\right]^{p^{\prime}} v_{i, j}\right)^{1 / p^{\prime}}
\end{aligned}
$$

where $a=\left\{a_{i, j}\right\}_{i, j=1}^{\infty}$ is decreasing in both $i$ and $j$.

Proof Denote the left side of (4.1) by $O(b)$. To prove the lower bound, suppose $\left\{\alpha_{n, m}\right\}_{n, m=1}^{\infty}$ is an arbitrary sequence $\alpha_{n, m} \geq 0, n, m \in \mathbb{N}$ and let

$$
a_{i, j}=\sum_{n=i}^{\infty} \sum_{m=j}^{\infty} \alpha_{n, m}
$$


Then

$$
\begin{aligned}
O(b) & \geq \sup _{a_{i, j}=\sum_{i} \sum_{j} \alpha_{n, m}} \frac{\sum_{i, j=1}^{\infty} a_{i, j} b_{i, j}}{\left(\sum_{i, j=1}^{\infty} a_{i, j}^{p} v_{i, j}\right)^{1 / p}} \\
& =\sup _{\alpha_{n, m} \geq 0} \frac{\sum_{i, j=1}^{\infty} b_{i, j}\left(\sum_{n=i}^{\infty} \sum_{m=j}^{\infty} \alpha_{n, m}\right)}{\left\{\sum_{i, j=1}^{\infty} v_{i, j}\left(\sum_{n=i}^{\infty} \sum_{m=j}^{\infty} \alpha_{n, m}\right)^{p}\right\}^{1 / p}} .
\end{aligned}
$$

But by (2.10) of Corollary 2.6 and an interchange of order of summation, it follows that

$O(b)$

$$
\begin{aligned}
& \geq C^{-1 / p} \sup _{\alpha_{n, m} \geq 0} \frac{\sum_{n=1}^{\infty} \sum_{m=1}^{\infty}\left(\alpha_{n, m} V_{n, m} / v_{n, m}\right)\left(v_{n, m} / V_{n, m}\right) \sum_{i=1}^{n} \sum_{j=1}^{m} b_{i, j}}{\left\{\sum_{i=1}^{\infty} \sum_{j=1}^{\infty}\left(\alpha_{i, j} V_{i, j} / v_{i, j}\right)^{p} v_{i, j}\right\}^{1 / p}} \\
& =C^{-1 / p}\left\{\sum_{n=1}^{\infty} \sum_{m=1}^{\infty}\left[\frac{1}{V_{n, m}} \sum_{i=1}^{n} \sum_{j=1}^{m} b_{i, j}\right]^{p^{\prime}} v_{n, m}\right\}^{1 / p^{\prime}}
\end{aligned}
$$

where the last equality follows from Theorem 2.4. Since $V_{n, m}=V_{n}^{\prime} V_{m}^{\prime \prime}$, the lower bound follows.

To prove the upper bound of $O(b)$, we proceed as in the continuous case. The interchange of order of summation and the monotonicity of $\left\{a_{i, j}\right\}_{i, j=1}^{\infty}$ shows that

$$
\begin{aligned}
\sum_{i, j=1}^{\infty} a_{i, j} b_{i, j} & =\sum_{j=1}^{\infty}\left[\sum_{i=1}^{\infty} a_{i, j} b_{i, j}\left(V_{i}^{\prime}\right)^{-1} \sum_{k=1}^{i} v_{k}^{\prime}\right] \\
& =\sum_{j=1}^{\infty}\left[\sum_{k=1}^{\infty} v_{k}^{\prime} \sum_{i=k}^{\infty} a_{i, j} b_{i, j}\left(V_{i}^{\prime}\right)^{-1}\right] \\
& \leq \sum_{j=1}^{\infty}\left[\sum_{k=1}^{\infty} v_{k}^{\prime} a_{k, j} \sum_{i=k}^{\infty} b_{i, j}\left(V_{i}^{\prime}\right)^{-1}\right]
\end{aligned}
$$

If $B_{k, j}=\sum_{i=1}^{k} b_{i, j}, B_{0, j}=0, j \in \mathbb{N}$ then, since $V_{\infty}^{\prime}=\infty$, Abel's formula (2.14) shows that

$$
\sum_{i=k}^{\infty} b_{i, j}\left(V_{i}^{\prime}\right)^{-1} \leq \sum_{i=k}^{\infty} B_{i, j} v_{i+1}^{\prime}\left(V_{i}^{\prime}\right)^{-1}\left(V_{i+1}^{\prime}\right)^{-1},
$$


so that

$$
\begin{aligned}
\sum_{i, j=1}^{\infty} a_{i, j} b_{i, j} & \leq \sum_{j=1}^{\infty}\left[\sum_{k=1}^{\infty} v_{k}^{\prime} a_{k, j} \sum_{i=k}^{\infty} B_{i, j} v_{i+1}^{\prime}\left(V_{i}^{\prime}\right)^{-1}\left(V_{i+1}^{\prime}\right)^{-1}\right] \\
& =\sum_{j=1}^{\infty} \sum_{k=1}^{\infty} v_{k}^{\prime} a_{k, j} U_{k, j} \\
& =\sum_{k=1}^{\infty} v_{k}^{\prime} \sum_{j=1}^{\infty} a_{k, j} U_{k, j}\left(V_{j}^{\prime \prime}\right)^{-1} \sum_{s=1}^{j} v_{s}^{\prime \prime}
\end{aligned}
$$

where $U_{k, j}=\sum_{i=k}^{\infty} B_{i, j} v_{i+1}^{\prime}\left(V_{i}^{\prime}\right)^{-1}\left(V_{i+1}^{\prime}\right)^{-1}$. Again an interchange of order of summation and the fact that $a_{k, j}$ is decreasing in $j$ shows that

$$
\begin{aligned}
\sum_{j=1}^{\infty} a_{k, j} U_{k, j}\left(V_{j}^{\prime \prime}\right)^{-1} \sum_{s=1}^{j} v_{s}^{\prime \prime} & =\sum_{s=1}^{\infty} v_{s}^{\prime \prime} \sum_{j=1}^{\infty} a_{k, j} U_{k, j}\left(V_{j}^{\prime \prime}\right)^{-1} \\
& \leq \sum_{s=1}^{\infty} v_{s}^{\prime \prime} a_{k, s} \sum_{j=s}^{\infty} U_{k, j}\left(V_{j}^{\prime \prime}\right)^{-1}
\end{aligned}
$$

But if $W_{k, n}=\sum_{s=1}^{n} U_{k, j}, W_{k, 0}=0, k, n \in \mathbb{N}$ then again by Abel's formula (2.14)

$$
\sum_{j=s}^{\infty} U_{k, j}\left(V_{j}^{\prime \prime}\right)^{-1} \leq \sum_{j=s}^{\infty} W_{k, j} v_{j+1}^{\prime \prime}\left(V_{j}^{\prime \prime}\right)^{-1}\left(V_{j+1}^{\prime \prime}\right)^{-1}
$$

Substituting into (4.3) and then the result into (4.2) shows that

$$
\begin{aligned}
\sum_{i, j=1}^{\infty} a_{i, j} b_{i, j} & \leq \sum_{k=1}^{\infty} v_{k}^{\prime} \sum_{s=1}^{\infty} v_{s}^{\prime \prime} a_{k, s} \sum_{j=s}^{\infty} W_{k, j} v_{j+1}^{\prime \prime}\left(V_{j}^{\prime \prime}\right)^{-1}\left(V_{j+1}^{\prime \prime}\right)^{-1} \\
& \leq \sum_{k, s=1}^{\infty} a_{k, s} v_{k, s} \sum_{j=s}^{\infty} W_{k, j}\left(V_{j}^{\prime \prime}\right)^{-1} \\
& \leq\left(\sum_{k, s=1}^{\infty} a_{k, s}^{p} v_{k, s}\right)^{1 / p}\left(\sum_{k, s=1}^{\infty} v_{k, s}\left(\sum_{j=1}^{\infty} W_{k, j}\left(V_{j}^{\prime \prime}\right)^{-1}\right)^{p^{\prime}}\right)^{1 / p^{\prime}}
\end{aligned}
$$


by Hölder's inequality and the fact that $v_{j+1}^{\prime \prime}\left(V_{j+1}^{\prime \prime}\right)^{-1} \leq 1$. Also since $v_{i+1}^{\prime}\left(V_{i+1}^{\prime}\right)^{-1} \leq 1$ then by the definition of $W_{k, j}$

$$
\begin{aligned}
W_{k, j} & =\sum_{\ell=1}^{j} U_{k, \ell} \\
& =\sum_{\ell=1}^{j} \sum_{i=k}^{\infty} B_{i, \ell} v_{i+1}^{\prime}\left(V_{i}^{\prime}\right)^{-1}\left(V_{i+1}^{\prime}\right)^{-1} \\
& \leq \sum_{i=k}^{\infty}\left(V_{i}^{\prime}\right)^{-1} \sum_{\ell=1}^{j} B_{i, \ell}=\sum_{i=k}^{\infty}\left(V_{i}^{\prime}\right)^{-1} \sum_{\ell=1}^{j} \sum_{\alpha=1}^{i} b_{\alpha, \ell} .
\end{aligned}
$$

Hence the second product of (4.4) is not larger than

$$
\begin{gathered}
\left\{\sum_{k, s=1}^{\infty} v_{k, s}\left[\sum_{j=s}^{\infty} \sum_{i=k}^{\infty}\left(V_{j}^{\prime \prime}\right)^{-1}\left(V_{i}^{\prime}\right)^{-1} \sum_{\alpha=1}^{i} \sum_{\ell=1}^{j} b_{\alpha, \ell}\right]^{p^{\prime}}\right\}^{1 / p^{\prime}} \\
\leq C\left\{\sum_{i, j=1}^{\infty} v_{i, j}\left(V_{i, j}^{-1}\right)^{p^{\prime}}\left(\sum_{\alpha=1}^{i} \sum_{\ell=1}^{j} b_{\alpha, \ell}\right)^{p^{\prime}}\right\}^{1 / p^{\prime}}
\end{gathered}
$$

by (2.10) of Corollary 2.6. Hence, according to (4.4)

$$
\frac{\sum_{i, j=1}^{\infty} a_{i, j} b_{i, j}}{\left(\sum_{k, s=1}^{\infty} a_{k, s}^{p} v_{k, s}\right)^{1 / p}} \leq C\left\{\sum_{i, j=1}^{\infty}\left[\frac{1}{V_{i}^{\prime} V_{j}^{\prime \prime}} \sum_{\alpha=1}^{i} \sum_{\ell=1}^{j} b_{\alpha, \ell}\right]^{p^{\prime}} v_{i, j}\right\}^{1 / p^{\prime}}
$$

and thus the upper bound of $O(b)$ follows.

Remark 4.2

(a) If the sequence $\left\{a_{i, j}\right\}_{i, j=1}^{\infty}$ is increasing in both $i$ and $j$ or decreasing in $i$ and increasing in $j$ duality theorems similar to that of Theorem 4.1 can be proved. However, unlike the continuous case (Corollaries 3.3 and 3.4) the result cannot be obtained from a change of index (variable), but one requires corresponding results to Corollary 2.6. We shall not give the details.

(b) If in (4.1) we take $b_{i, j}=u_{i, j}, i, j \in \mathbb{N}$ then the upper bound of (4.1) yields the inequality

$$
\sum_{i, j=1}^{\infty} a_{i, j} u_{i, j} \leq C\left(\sum_{i, j=1}^{\infty} a_{i, j}^{p} v_{i, j}\right)^{1 / p}
$$


where $0 \leq a \Downarrow$, provided the right side of (4.1) (with $b_{i, j}=u_{i, j}$ ) is finite.

Finally, the proof of Theorem 4.1 also yields the one dimensional result:

Corollary 4.3 If $\left\{a_{i}\right\}_{i=1}^{\infty}$ is a non-negative decreasing sequence and $0 \leq b=\left\{b_{i}\right\}_{i=1}^{\infty}$ then for $1<p<\infty$,

$$
\sup _{0 \leq a \Downarrow} \frac{\sum_{i=1}^{\infty} a_{i} b_{i}}{\left(\sum_{i=1}^{\infty} a_{i}^{p} v_{i}\right)^{1 / p}} \approx\left[\sum_{i=1}^{\infty}\left(\frac{1}{V_{i}} \sum_{k=1}^{i} b_{k}\right)^{p^{\prime}} v_{i}\right]^{1 / p^{\prime}},
$$

where $V_{i}=\sum_{k=1}^{i} v_{k}$ satisfies $V_{\infty}=\infty$.

This result was proved in $[9$, p. 368] under the additional condition that $\lim _{n \rightarrow \infty}\left(v_{n+1} / v_{n}\right)=C>0$.

\section{APPLICATIONS}

Suppose $T$ is a positive integral operator defined on functions $f: \mathbb{R}_{+}^{n} \rightarrow \mathbb{R}_{+}$, decreasing in each of its variables $(0 \leq f \Downarrow)$ and $T^{*}$ its formal adjoint. If $v$ is a product weight such that $V_{i}\left(x_{i}\right)=\int_{0}^{x_{i}} v_{i}$ satisfies $V_{i}(\infty)=\infty, i=1,2, \ldots, n$ and $u$ any weight function, then Corollary 3.2 and Theorem 2.4 shows that the inequality

$$
\left(\int_{\mathbb{R}_{+}^{n}}|T f(x)|^{q} u(x) d x\right)^{1 / q} \leq C\left(\int_{\mathbb{R}_{+}^{n}} f(x)^{p} v(x) d x\right)^{1 / p}
$$

$1<p, q<\infty, 0 \leq f \Downarrow$, is equivalent to the inequality

$$
\begin{gathered}
\left(\int_{\mathbb{R}_{+}^{n}}\left(\int_{0}^{x} T^{*} g\right)^{p^{\prime}} V(x)^{-p^{\prime}} v(x) d x\right)^{1 / p^{\prime}} \\
\leq C\left(\int_{\mathbb{R}_{+}^{n}} g(x)^{q^{\prime}} u(x)^{1-q^{\prime}} d x\right)^{1 / q^{\prime}}
\end{gathered}
$$

where $0 \leq g$, is arbitrary. 
Hence, to characterize the weights $u, v$ for which (5.1) is satisfied (with $0 \leq f \Downarrow$ ) is equivalent to characterize the weights for which (5.2) is satisfied. In other words the characterization of weights for which (5.1) holds is equivalent to characterize the weights for which $T_{1}$ : $L_{u^{1-q^{\prime}}}^{q^{\prime}} \rightarrow L_{V^{-p^{\prime} v}}^{p^{\prime}}$ is bounded, where

$$
\left(T_{1} g\right)(x)=\int_{0}^{x} T^{*} g
$$

Similar notions result from our duality theorems in Section 3 when $T$ is defined on the cone of functions which are monotone (increasing, decreasing or increasing in some variables and decreasing in others).

Of course it still may be a formidable task to characterize weight functions for which (5.2) is satisfied for general $T$. However, for some operators this is possible and if $u$ is in addition also a product weight then some results are quite simple.

The simplest case is when $T$ is the identity operator. In fact, Theorem 3.1 implies the following somewhat weaker version of a recent result in [2, Theorem 2.2, Theorem 2.5]:

TheOREm 5.1 Suppose $u$ and $v$ are product weights on $\mathbb{R}_{+}^{n}$ and $1<p \leq$ $q<\infty$.

(a) If $V(x)=\int_{0}^{x} v, V_{i}\left(x_{i}\right)=\int_{0}^{x_{i}} v_{i}$ with $V_{i}(\infty)=\infty, i=1, \ldots, n$, then the following are equivalent:

(i) For $0 \leq f \Downarrow$

$$
\left(\int_{\mathbb{R}_{+}^{n}} f^{q} u\right)^{1 / q} \leq C\left(\int_{\mathbb{R}_{+}^{n}} f^{p} v\right)^{1 / p}
$$

is satisfied.

(ii) For any $g \geq 0$

$$
\begin{aligned}
& \left(\int_{\mathbb{R}^{n}}\left(\int_{0}^{x} g\right)^{p^{\prime}} V(x)^{-p^{\prime}} v(x) d x\right)^{1 / p^{\prime}} \\
& \quad \leq C\left(\int_{\mathbb{R}_{+}^{n}} g(x)^{q^{\prime}} u(x)^{1-q^{\prime}} d x\right)^{1 / q^{\prime}}
\end{aligned}
$$

holds 
(iii) $\sup _{s>0}\left(\int_{0}^{s} v_{i}(t) d t\right)^{-1 / p}\left(\int_{0}^{s} u_{i}(t) d t\right)^{1 / q}<\infty$ for $i=1, \ldots, n$.

(b) If $V_{i}^{*}\left(x_{i}\right)=\int_{x_{i}}^{\infty} v_{i}$ satisfies $V_{i}^{*}(0)=\infty, i=1, \ldots, n, V^{*}(x)=\int_{x}^{\infty} v$ then for $0 \leq f \Uparrow,(5.3)$ is equivalent to (5.4) with $V$ replaced by $V^{*}$, which in turn is equivalent to

$$
\sup _{s>0}\left(\int_{s}^{\infty} v_{i}\right)^{-1 / p}\left(\int_{s}^{\infty} u_{i}\right)^{1 / q}<\infty
$$

$i=1, \ldots, n$.

Proof (a) We already noted the equivalence of (i) and (ii). That (ii) and (iii) are equivalent follows from Theorem 2.1 with $f$ replaced by $g, q$ by $p^{\prime}, u$ by $v V^{-p^{\prime}}$ and $v$ by $u^{1-q^{\prime}}$. Then by (2.2), (5.4) is equivalent to

$$
\begin{aligned}
\sup _{s>0} & \left(\int_{s}^{\infty} v_{i}(t) V_{i}(t)^{-p^{\prime}} d t\right)^{1 / p^{\prime}}\left(\int_{0}^{s} u_{t}(t)^{\left(1-q^{\prime}\right)(1-q)} d t\right)^{1 / q} \\
= & \sup _{s>0} \frac{V_{i}(s)^{-1 / p}}{\left(p^{\prime}-1\right)^{1 / p^{\prime}}}\left(\int_{0}^{s} u_{i}(t) d t\right)^{1 / q}<\infty
\end{aligned}
$$

$i=1, \ldots, n$.

The proof of (b) follows similarly - or alternately from (a) via changes of variables.

Remark 5.2 Recall (cf. [5]) that if $f$ is a measurable function on $(X, \mu)$, then $\lambda_{f}(\alpha)=\mu(\{x \in X:|f(x)|>\alpha\}), \alpha>0$, is the distribution function of $f$ and its generalized inverse, or more precisely $f^{*}(t)=\inf _{\alpha>0}\left\{\lambda_{f}(\alpha)\right.$ $\leq t\}$ is the decreasing rearrangement of $|f|$. It is clear that by Fubini's theorem if $p>0$

$$
\int_{X}|f|^{p} d \mu=\int_{0}^{\infty} f^{*}(t)^{p} d t
$$

If $\omega$ is a weight function and the Lorentz spaces are defined by

$$
\Lambda_{p, \omega}=\left\{f:\left(\int_{0}^{\infty} f^{*}(t)^{p} \omega(t) d t\right)^{1 / p}<\infty\right\}
$$


then the one dimensional form of Theorem 5.1 shows that for $1<p \leq q<\infty, \Lambda_{p, v} \subset \Lambda_{q, u}$ if and only if $u, v$ satisfy (iii) with $i=1$.

In [1], Barza has shown that one may extend the rearrangement of a function $f(x, y)$, where $(x, y) \in(X \times Y)$ and $(X, \mu)(Y, \mu)$ are measure spaces. For fixed $x$ write $f(x, y)=f_{x}(y)$ and let $f_{x}^{*}(t)$ be its rearrangement with respect to $y$. If $f_{x}^{*}(t)=F_{t}(x)$ then let $F_{t}^{*}(s)$ be the rearrangement of $F$ with respect to $x$ for fixed $y$. We denote $F_{t}^{*}(s)=f_{21}^{*}(s, t)$, where the subscript denotes that one rearranged $f$ with respect to the second variable first, and then with the first variable.

It is not difficult to see that $f_{21}^{*} \neq f_{12}^{*}$, however

$$
\int_{X}\left(\int_{Y}|f| d \mu\right) d \mu=\int_{0}^{\infty} \int_{0}^{\infty} f_{21}^{*}(s, t) d t d s=\int_{0}^{\infty} \int_{0}^{\infty} f_{12}^{*}(s, t) d t d s
$$

In analogy to the Lorentz spaces defined above one can define the "two-dimensional" Lorentz spaces by

$$
\begin{aligned}
\Lambda_{p, \omega}^{2}= & \{f \text { defined on }(X \times Y, \mu \times \mu): \\
& \left.\left(\int_{0}^{\infty} \int_{0}^{\infty} f_{2,1}^{*}(s, t)^{p} \omega(s, t) d t d s\right)^{1 / p}<\infty\right\},
\end{aligned}
$$

where $0<p<\infty$ and $\omega$ a weight on $\mathbb{R}_{+}^{2}$. Hence again, Theorem 5.1 with $n=2$, shows that for product weights $u$ and $v$

$$
\Lambda_{p, v}^{2} \subset \Lambda_{q, u}^{2}, \quad 1<p \leq q<\infty
$$

if and only if (iii) holds.

The next result concerns the two dimensional Hardy operator, defined on decreasing function.

THEOREM 5.3 Suppose $u$ and $v$ are product weights, $V_{i}\left(x_{i}\right)=$ $\int_{0}^{x_{i}} v_{i}(t) d t, i=1,2$; satisfies $V_{i}(\infty)=\infty$. If $1<p \leq q<\infty$ and $0 \leq f \Downarrow$, 
then

$$
\left(\int_{\mathbb{R}_{+}^{2}}\left(\int_{0}^{x} f\right)^{q} u(x) d x\right)^{1 / q} \leq C\left(\int_{\mathbb{R}_{+}^{2}} f^{p}(x) v(x) d x\right)^{1 / p}
$$

is satisfied, if and only if for $i=1,2$

$$
\sup _{s>0}\left(\int_{0}^{s} v_{i}(t) d t\right)^{-1 / p}\left(\int_{0}^{s} u_{i}(t) t^{q} d t\right)^{1 / q}<\infty
$$

and

$$
\sup _{s>0}\left(\int_{0}^{s} t^{p^{\prime}} V_{i}(t)^{-p^{\prime}} v_{i}(t) d t\right)^{1 / p^{\prime}}\left(\int_{s}^{\infty} u_{i}(t) d t\right)^{1 / q}<\infty
$$

Proof Since $T f(x)=\int_{0}^{x} f$ its conjugate is $T^{*} g(x)=\int_{x}^{\infty} g$. But (5.5) is equivalent to (5.2) for arbitrary $g \geq 0$. Hence to characterize the weights for which (5.2) is satisfied, we must compute

$$
\begin{aligned}
\int_{0}^{x} T^{*} g & =\int_{0}^{x_{1}} \int_{0}^{x_{2}}\left(\int_{t_{1}}^{\infty} \int_{t_{2}}^{\infty} g\left(\alpha_{1}, \alpha_{2}\right) d \alpha_{2} d \alpha_{1}\right) d t_{2} d t_{1} \\
& =\int_{0}^{x_{1}}\left[\int_{0}^{x_{2}}\left(\int_{t_{2}}^{\infty} G\left(t_{1}, \alpha_{2}\right) d \alpha_{2}\right) d t_{2}\right] d t_{1}
\end{aligned}
$$

where $G\left(t_{1}, \alpha_{2}\right)=\int_{t_{1}}^{\infty} g\left(\alpha_{1}, \alpha_{2}\right) d \alpha_{1}$. But since

$$
\begin{aligned}
\int_{0}^{x_{2}} & \left(\int_{t_{2}}^{\infty} G\left(t_{1}, \alpha_{2}\right) d \alpha_{2}\right) d t_{2} \\
= & \int_{0}^{x_{2}} \int_{t_{2}}^{x_{2}} G\left(t_{1}, \alpha_{2}\right) d \alpha_{2} d t_{2}+x_{2} \int_{x_{2}}^{\infty} G\left(t_{1}, \alpha_{2}\right) d \alpha_{2} \\
= & \int_{0}^{x_{2}} \alpha_{2} G\left(t_{1}, \alpha_{2}\right) d \alpha_{2}+x_{2} \int_{x_{2}}^{\infty} G\left(t_{1}, \alpha_{2}\right) d \alpha_{2}
\end{aligned}
$$


it follows on interchanging the order of integration that

$$
\begin{aligned}
\int_{0}^{x} T^{*} g= & \int_{0}^{x_{2}} \int_{0}^{x_{1}} \alpha_{2} G\left(t_{1}, \alpha_{2}\right) d t_{1} d \alpha_{2} \\
& +x_{2} \int_{0}^{x_{1}} \int_{x_{2}}^{\infty} G\left(t_{1}, \alpha_{2}\right) d \alpha_{2} d t_{1} \\
= & \int_{0}^{x_{2}} \alpha_{2} \int_{0}^{x_{1}}\left(\int_{t_{1}}^{\infty} g\left(\alpha_{1}, \alpha_{2}\right) d \alpha_{1}\right) d t_{1} d \alpha_{2} \\
& +x_{2} \int_{x_{2}}^{\infty} \int_{0}^{x_{1}}\left(\int_{t_{1}}^{\infty} g\left(\alpha_{1}, \alpha_{2}\right) d \alpha_{1}\right) d t_{1} d \alpha_{2} \\
= & \int_{0}^{x_{2}} \alpha_{2} \int_{0}^{x_{1}}\left(\int_{t_{1}}^{x_{1}} g\left(\alpha_{1}, \alpha_{2}\right) d \alpha_{1}\right) d t_{1} d \alpha_{2} \\
& +x_{1} \int_{0}^{x_{2}} \alpha_{2} \int_{x_{1}}^{\infty} g\left(\alpha_{1}, \alpha_{2}\right) d \alpha_{1} d \alpha_{2} \\
& +x_{2} \int_{x_{2}}^{\infty} \int_{0}^{x_{1}}\left(\int_{t_{1}}^{x_{1}} g\left(\alpha_{1}, \alpha_{2}\right) d \alpha_{1}\right) d t_{1} d \alpha_{2} \\
& +x_{2} x_{1} \int_{x_{2}}^{\infty} \int_{x_{1}}^{\infty} g\left(\alpha_{1}, \alpha_{2}\right) d \alpha_{1} d \alpha_{2} \\
= & \int_{0}^{x_{2}} \alpha_{2} \int_{0}^{x_{1}} \alpha_{1} g\left(\alpha_{1}, \alpha_{2}\right) d \alpha_{1} d \alpha_{2} \\
& +x_{1} \int_{0}^{x_{2}} \int_{x_{1}}^{\infty} \alpha_{2} g\left(\alpha_{1}, \alpha_{2}\right) d \alpha_{1} d \alpha_{2} \\
& +x_{2} \int_{x_{2}}^{\infty} \int_{0}^{x_{1}} \alpha_{1} g\left(\alpha_{1}, \alpha_{2}\right) d \alpha_{1} d \alpha_{2}+x_{1} x_{2} \int_{x_{1}}^{\infty} \int_{x_{2}}^{\infty} g \\
= & \int_{0}^{x} \alpha g(\alpha) d \alpha+x_{1} \int_{x_{1}}^{\infty} \int_{0}^{x_{2}} \alpha_{2} g(\alpha) d \alpha_{2} d \alpha_{1} \\
& +x_{2} \int_{0}^{x_{1}} \int_{x_{2}}^{\infty} \alpha_{1} g(\alpha) d \alpha+x \int_{x}^{\infty} g \\
= & I_{1}(x)+I_{2}(x)+I_{3}(x)+I_{4}(x), \\
& =x_{1}(x)
\end{aligned}
$$

respectively. Hence by Minkowski's inequality, (5.2) with $n=2$, will be satisfied, if and only if

$$
\begin{aligned}
& \left(\int_{\mathbb{R}_{+}^{2}} I_{j}(x)^{p^{\prime}} V(x)^{-p^{\prime}} v(x) d x\right)^{1 / p^{\prime}} \\
& \quad \leq C\left(\int_{\mathbb{R}_{+}^{2}} g^{q^{\prime}} u^{1-q^{\prime}}\right)^{1 / q^{\prime}} \equiv C J
\end{aligned}
$$


is satisfied for $j=1,2,3,4$. Now we apply Theorem 2.1 twice with $q=p^{\prime}$. With $f(\alpha)=\alpha g(\alpha)$

$$
\begin{gathered}
\left(\int_{\mathbb{R}_{+}^{2}} I_{1}(x)^{p^{\prime}} V(x)^{-p} v(x) d x\right)^{1 / p^{\prime}} \\
=\left(\int_{\mathbb{R}_{+}^{2}}\left(\int_{0}^{x} \alpha g(\alpha) d \alpha\right)^{p^{\prime}} V(x)^{-p^{\prime}} v(x) d x\right)^{1 / p^{\prime}} \\
\leq C\left(\int_{\mathbb{R}_{+}^{2}}(\alpha g(\alpha))^{q^{\prime}} \frac{u(\alpha)^{1-q^{\prime}}}{\alpha^{q^{\prime}}} d \alpha\right)^{1 / q^{\prime}}=C J
\end{gathered}
$$

if and only if

$$
\begin{aligned}
\sup _{s>0} & \left(\int_{s}^{\infty} v_{i}(t) V_{i}(t)^{-p^{\prime}} d t\right)^{1 / p^{\prime}}\left(\int_{0}^{s}\left(\frac{u_{i}(t)^{1-q^{\prime}}}{t^{q^{\prime}}}\right)^{1-q} d t\right)^{1 / q^{\prime}} \\
& =\sup _{s>0} \frac{1}{\left(p^{\prime}-1\right)^{1 / p^{\prime}}}\left(\int_{0}^{s} v_{i}(t) d t\right)^{-1 / p}\left(\int_{0}^{s} u_{i}(t) t^{q} d t\right)^{1 / q}<\infty .
\end{aligned}
$$

But this is clearly the case by (5.6). Also by Theorem 2.1

$$
\begin{aligned}
& \left(\int_{\mathbb{R}_{+}^{2}} I_{4}(x)^{p^{\prime}} V(x)^{-p^{\prime}} v(x) d x\right)^{1 / p^{\prime}} \\
& \quad=\left(\int_{\mathbb{R}_{+}^{2}} x^{p^{\prime}} V(x)^{-p^{\prime}} v(x)\left(\int_{x}^{\infty} g\right)^{p^{\prime}} d x\right)^{1 / p^{\prime}} \leq C J
\end{aligned}
$$

if and only if (5.7) is satisfied. Next, by Corollary 2.3

$$
\begin{aligned}
& \left(\int_{\mathbb{R}_{+}^{2}} I_{3}(x)^{p^{\prime}} V(x)^{-p^{\prime}} v(x) d x\right)^{1 / p^{\prime}} \\
& \quad=\left(\int_{\mathbb{R}_{+}^{2}} x_{2}^{p^{\prime}}\left(\int_{0}^{x_{1}} \int_{x_{2}}^{\infty} \alpha_{1} g(\alpha) d \alpha\right)^{p^{\prime}} V(x)^{-p^{\prime}} v(x) d x\right)^{1 / p^{\prime}} \\
& \quad \leq C\left(\int_{\mathbb{R}_{+}^{2}}\left(\alpha_{1} g(\alpha)\right)^{q^{\prime}} \frac{u(\alpha)^{1-q^{\prime}}}{\alpha_{1}^{q^{\prime}}} d \alpha\right)^{1 / q^{\prime}}=C J
\end{aligned}
$$


if and only if

$$
\begin{aligned}
\sup _{s>0} & \left(\int_{s}^{\infty} v_{1}(t) V_{1}(t)^{-p^{\prime}} d t\right)^{1 / p^{\prime}}\left(\int_{0}^{s} \frac{u_{1}(t)}{t^{-q}} d t\right)^{1 / q} \\
& =\sup _{s>0} \frac{1}{\left(p^{\prime}-1\right)^{1 / p^{\prime}}}\left(\int_{0}^{s} v_{1}(t) d t\right)^{-1 / p}\left(\int_{0}^{s} u_{1}(t) t^{q} d t\right)^{1 / q}<\infty
\end{aligned}
$$

and

$$
\sup _{s>0}\left(\int_{0}^{s} t^{p^{\prime}} V_{2}(t)^{-p^{\prime}} v_{2}(t) d t\right)^{1 / p^{\prime}}\left(\int_{s}^{\infty} u_{2}(t) d t\right)^{1 / q}<\infty .
$$

But this is the case by (5.6) with $i=1$ and (5.7) with $i=2$. By symmetry we obtain the estimate involving $I_{2}$ in the same way provided (5.6) holds with $i=2$ and (5.7) with $i=1$. This proves the theorem.

$$
\text { If } \quad 1<p \leq q<\infty, \quad v_{i}(t)=t^{\alpha_{i}}, \quad u_{i}(t)=t^{\beta_{i}-q}, \quad-1<\alpha_{i}<p-1,
$$
$-1<\beta_{i}<q-1$ and $\left(\alpha_{i}+1\right) a=\left(\beta_{i}+1\right) p, i=1,2$ is satisfied, then (5.6) and (5.7) are satisfied. Hence for the weights $v(x)=x_{1}^{\alpha_{1}} x_{2}^{\alpha_{2}}, u(x)=$ $x_{1}^{\beta_{1}-q} x_{2}^{\beta_{2}-q}$ Theorem 5.3 holds.

It is clear that Theorem 5.3 has a discrete analogue.

Consider sequences $0 \leq a=\left\{a_{i, j}\right\}_{i, j=1}^{\infty}, 0 \leq b=\left\{b_{i, j}\right\}_{i, j=1}^{\infty}$. Then the two dimensional Césaro operator and its conjugate Copson operator are defined by

$$
\sigma_{i, j}(a)=\sum_{k=1}^{i} \sum_{\ell=1}^{j} a_{k, \ell}, \quad \sigma_{i, j}^{*}(b)=\sum_{k=i}^{\infty} \sum_{\ell=j}^{\infty} b_{k, \ell} .
$$

Suppose $u=\left\{u_{i, j}\right\}_{i, j=1}^{\infty}, v=\left\{v_{i, j}\right\}_{i, j=1}^{\infty}$ are product sequences, that is $u_{i, j}=u_{1, i} u_{2, j}, v_{i, j}=v_{1, i} v_{2, j} i, j \in \mathbb{N}$. Denote also by $V_{1, i}=\sum_{k=1}^{i} v_{1, k}$, $V_{2, j}=\sum_{\ell=1}^{j} v_{2, \ell}$ then the discrete analogue of Theorem 5.3 has the form:

Theorem 5.4 Suppose $1<p \leq q<\infty, u=\left\{u_{i, j}\right\}_{i, j=1}^{\infty}, v=\left\{v_{i, j}\right\}_{i, j=1}^{\infty}$ are product sequences, such that $V_{1, \infty}=V_{2, \infty}=\infty$. Then the following are equivalent:

(i) For $0 \leq a \Downarrow$ (i.e., $\left\{a_{i, j}\right\}_{i, j=1}^{\infty}$ decreases in $i$ and $j$ )

$$
\left(\sum_{i, j=1}^{\infty} \sigma_{i, j}(a)^{q} u_{i, j}\right)^{1 / q} \leq C\left(\sum_{i, j=1}^{\infty} d_{i, j}^{p} v_{i, j}\right)^{1 / p}
$$

is satisfied. 
(ii) For $0 \leq b=\left\{b_{i, j}\right\}_{i, j=1}^{\infty}$ arbitrary,

$$
\begin{aligned}
& \left\{\sum_{i, j=1}^{\infty}\left(\sum_{k=1}^{i} \sum_{\ell=1}^{j} \sigma_{k, \ell}^{*}(b)\right)^{p^{\prime}}\left(V_{1, i} V_{2, j}\right)^{-p^{\prime}} v_{i, j}\right\}^{1 / p^{\prime}} \\
& \leq C\left\{\sum_{i, j=1}^{\infty} b_{i, j}^{q^{\prime}} u_{i, j}^{1-q^{\prime}}\right\}^{1 / q^{\prime}}
\end{aligned}
$$

holds.

(iii)

$$
\begin{aligned}
& \sup _{s \in \mathbb{N}}\left(\sum_{k=s}^{\infty} v_{\gamma, k}\left(V_{\gamma, k}\right)^{-p^{\prime}}\right)^{1 / p^{\prime}}\left(\sum_{k=1}^{s} u_{\gamma, k} k^{+q}\right)^{1 / q}<\infty \\
& \sup _{s \in \mathbb{N}}\left(\sum_{k=1}^{s} k^{p^{\prime}}\left(V_{\gamma, k}\right)^{-p^{\prime}} v_{\gamma, k}\right)^{1 / p^{\prime}}\left(\sum_{k=s}^{\infty} u_{\gamma, k}\right)^{1 / q}<\infty
\end{aligned}
$$

are satisfied for $\gamma=1$ and $\gamma=2$.

The proof is essentially the same as that of Theorem 5.3. The equivalence of (i) and (ii) follows from Theorem 4.1 and Theorem 2.4. The equivalence of (ii) and (iii) follows from estimates such as Corollary 2.6 obtained from Theorem 2.5 once it is observed that

$$
\begin{aligned}
\sum_{k=1}^{i} \sum_{\ell=1}^{j} \sigma_{k, \ell}^{*}(b)= & \sum_{\alpha=1}^{i} \sum_{\beta=1}^{j} \alpha \beta b_{\alpha, \beta}+i \sum_{\alpha=1}^{\infty} \sum_{\beta=1}^{j} \beta b_{\alpha, \beta} \\
& +j \sum_{\alpha=1}^{i} \sum_{\beta=j}^{\infty} \alpha b_{\alpha, \beta}+i j \sum_{\alpha=i}^{\infty} \sum_{\beta=j}^{\infty} b_{\alpha, \beta} .
\end{aligned}
$$

We omit the details.

Note that the first supremum of (iii) does not have the form (5.6) because we cannot integrate. However, it follows from (2.13) that

$$
\left(\sum_{k=1}^{s} v_{\gamma, k}\right)^{-1 / p^{\prime}}\left(\sum_{k=1}^{s} u_{\gamma, k} k^{q}\right)^{1 / q} \leq C
$$

for all $x \in \mathbb{N}, \gamma=1,2$, implies that the finiteness of the first supremum in (iii). 


\section{References}

[1] Barza, S., Weighted multidimensional integral inequalities and applications, Doctoral Thesis, Luleå Univ. of Techn., Luleå, Sweden, December, 1999.

[2] Barza, S., Persson, L.-E. and Soria, J. (2000). Sharp weighted multidimensional integral inequalities for monotone functions, Math. Nachr., 210, 43-58.

[3] Barza, S., Persson, L.-E. and Stepanov, V. D. (2001). On weighted multidimensional embeddings for monotone functions, Math. Scand., 88.

[4] Bennett, G. (1991). Some elementary inequalities III, Quart. J. Math., Oxford Ser. (2), 42, 149-174.

[5] Bennett, C. and Sharpley, R., Interpolation of Operators, Acad. Press, 1988.

[6] Heinig, H. P. and Kufner, A. (1996). Hardy operators of monotone functions and sequences in Orlicz spaces, J. London Math. Soc. (2), 53, 256-270.

[7] Opic, B. and Kufner, A., Hardy-type inequalities, Pitman Research Notes in Math., Longman, Harlow, 1990.

[8] Sawyer, E. (1990). Boundedness of classical operators on classical Lorentz spaces, Studia Math., 96, 145-158.

[9] Sinnamon, G. (1994). Spaces defined by their level functions and their duals, Studia Math., 111(1), 19-52.

[10] Stepanov, V. D. (1993). Integral operators on the cone of monotone functions, J. London Math. Soc. (2), 48, 465-487. 